\title{
Myocardial Force-Velocity Relations Studied in Intact Unanesthetized Man*
}

\author{
Gerald Glick, $\dagger$ Edmund H. Sonnenblick, and Eugene Braunwald \\ (From the Cardiology Branch, National Heart Institute, Bethesda, Md.)
}

In 1938, A. V. Hill demonstrated that the inverse relationship between the force generated and the velocity of shortening constitutes one of the most fundamental mechanical properties of skeletal muscle (1). More recently it has been shown that a similar reciprocal relationship between force and velocity also exists in the isolated cat papillary muscle (2-5). Moreover, at any given muscle length, various positive inotropic interventions, such as augmentation of heart rate $(2,3)$, administration of norepinephrine $(3,4)$, $\mathrm{Ca}^{++}(3)$, or digitalis (6), shift the force-velocity relation so that the velocity of shortening is greater at any given load and the maximal velocity of the unloaded muscle is increased. This type of shift in the force-velocity relation is interpreted to reflect an augmentation of the contractile state of the myocardium. The ability to alter the relationship between the force generated and the velocity of shortening in heart muscle is in contrast with the relative stability of the forcevelocity relation in skeletal muscle.

Recently, studies on isolated human papillary muscle extended to man the concept that the force-velocity relation may be used to characterize the basic contractile state of heart muscle (6). Fry, Griggs, and Greenfield (7) and Levine and Britman (8) have shown in open-chest dogs that the inverse relationship between force and instantaneous velocity is applicable to the intact canine ventricle. The objective of the present investigation was to determine whether or not the intact ventricle in conscious human subjects adheres to this basic law of muscular contraction that had been elucidated in various experimental

* Submitted for publication December 28, 1964; accepted February 25, 1965.

Presented in part before the American Society for Clinical Investigation, May 4, 1964, Atlantic City, N. J.

$\dagger$ Established Investigator, American Heart Association. Address requests for reprints to Dr. Gerald Glick, National Heart Institute, Bethesda, Md. 20014. preparations, and whether analysis of the forcevelocity relation can be useful in assessing the effects of various physiologic and pharmacologic interventions on the human heart.

\section{Methods}

Thirteen patients, seven men and six women, who ranged in age from 16 to 55 years were studied (Table I). At the time of corrective cardiac operations small silver-tantalum markers had been sutured to the right ventricle, left ventricle, or both ventricles, as described in detail previously (9). Three patients underwent closure of an atrial septal defect and two patients closure of a ventricular septal defect; in two patients pulmonary valvulotomies had been performed for relief of pulmonic stenosis; four patients had mitral commissurotomies; one patient with aortic stenosis had his aortic valve replaced with a Starr-Edwards prosthesis, and one patient with mitral regurgitation and associated idiopathic complete atrioventricular block underwent replacement of the mitral valve, also with a Starr-Edwards prosthesis. As a result of these operations the major anatomic and physiologic abnormalities had been corrected, and at the time of study none of the patients had clinically significant limitations of cardiac reserve. The postoperative hemodynamic data obtained at cardiac catheterization, carried out 2 weeks to 17 months postoperatively, are shown in Table $I$. The resting cardiac outputs were within normal limits $\left(>2.50 \mathrm{~L}\right.$ per minute $\left.\mathrm{m}^{2}\right)$ in nine patients and just below this value in two patients (R.A. and I.D.). The mean pressures in both atria were within normal limits in all the patients who were studied, although the right and left ventricular end-diastolic pressures were slightly elevated in patient A.P. The pulmonary arterial pressures were within normal limits in all but three patients, in whom there was a slight elevation.

In order to obtain the data necessary for evaluation of the force-velocity relation, cineradiograms were exposed at 30 frames per second, while intraventricular pressure pulses were recorded simultaneously at a paper speed of $100 \mathrm{~mm}$ per second. Pressures were recorded through cardiac catheters by means of Statham P23D strain gauge pressure transducers. A mechanical marker was activated by the $R$ wave of the electrocardiogram, and its movement was recorded both on the cineradiogram and on the pressure tracing, thereby allowing precise temporal correlation of ventricular dimensions and pressures. Subsequently, the distances between markers 


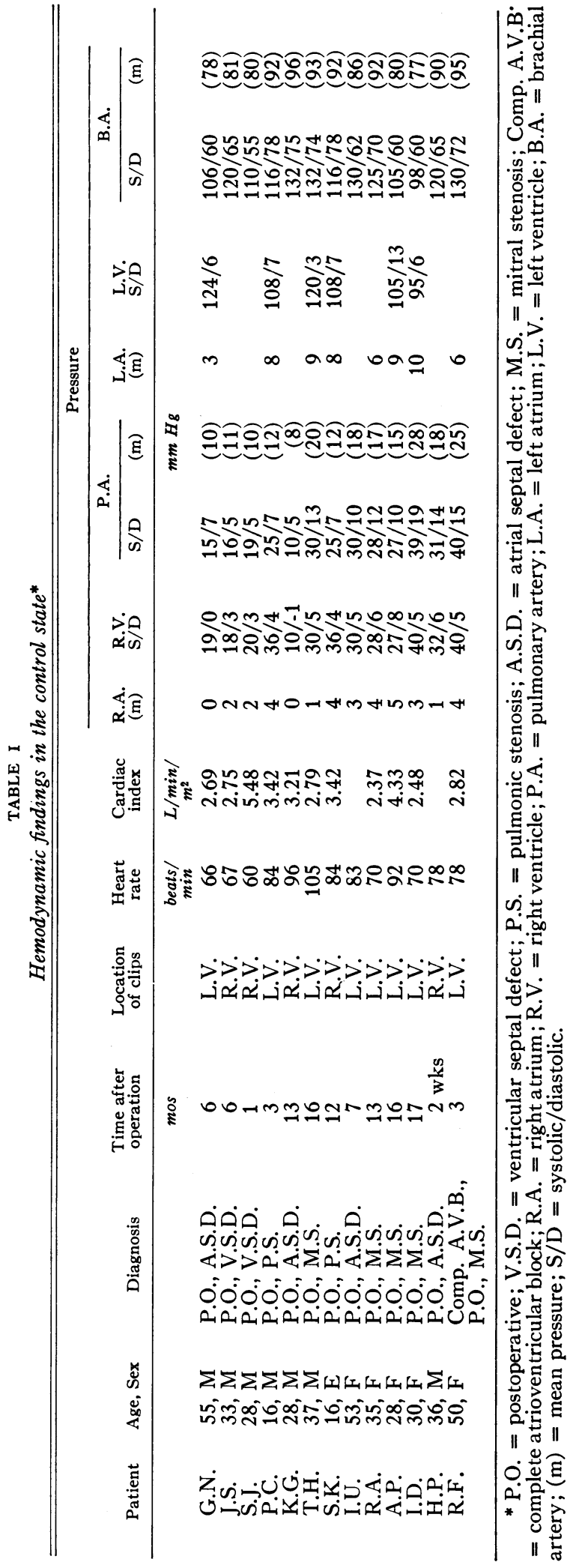

were measured on successive frames of the cineradiogram. The only ventricular dimensions measured were those between markers that had been placed in a manner so that the line that joined them was parallel, or nearly parallel, to the frontal plane, thereby minimizing the error that could result from rotation of the heart within the thorax during the cardiac cycle (9). The position of the markers in relation to the frontal plane was always determined by means of films exposed in the lateral projection. On the right ventricle, the distances measured were between two markers placed on the anterior surface, one just beneath the pulmonic valve, and the other at the most inferior portion of the anterior surface. On the left ventricle, the distances measured were between two markers placed along the lateral surface, one near its apex and the other near its base. All observations were made with the patient in the supine position, and in order to eliminate the effect of respiration on ventricular dimensions (10), all measurements were performed in end-expiration.

From analyses of force-velocity relationships in the isolated cat papillary muscle, it is clear that throughout the duration of the active state the position of the forcevelocity curve, at any instant, is determined by both the instantaneous length of the muscle and by its contractile state (11). The contractile state is defined by the curve relating velocity of shortening and force at any one muscle length. Therefore, if the force-velocity relation is examined at a single instant during contraction, and always at the same muscle length, then the contractile state of the myocardium will uniquely determine the position of the force-velocity curve for that particular muscle length. Accordingly, the basic plan in this investigation was to determine the force and velocity of a segment of the patient's ventricular myocardium when the myocardial segment passed through a specific length. Then the effects of a number of interventions on these two variablesforce and velocity-could be defined on a beat-to-beat basis at the same length of the segment of myocardium (Figure 1). This length of myocardium at which all measurements were carried out has been termed the "isolength point."

The analysis was accomplished in the following manner: First the instantaneous distances between the roentgenopaque markers were plotted below the intraventricular pressure pulses of the same beats, both for the control measurement and for the intervention. This distance between markers could be reproducibly measured to 0.5 $\mathrm{mm}$. The isolength point was then chosen by selecting a ventricular length that was common to all the cardiac cycles that were analyzed, and which occurred during the first two-thirds of the ejection period, before the active state of the muscle had declined. To obviate the effects of muscle length on the velocity of shortening $(3,4,11)$, the rate at which the markers approached each other was determined when the length of the segment of myocardium between them passed through the isolength point (Figure 1 , points $a$ and $\left.a^{\prime}\right)$. The instantaneous velocity of shortening at the time the distance between the markers passed through the isolength point was determined by measuring 


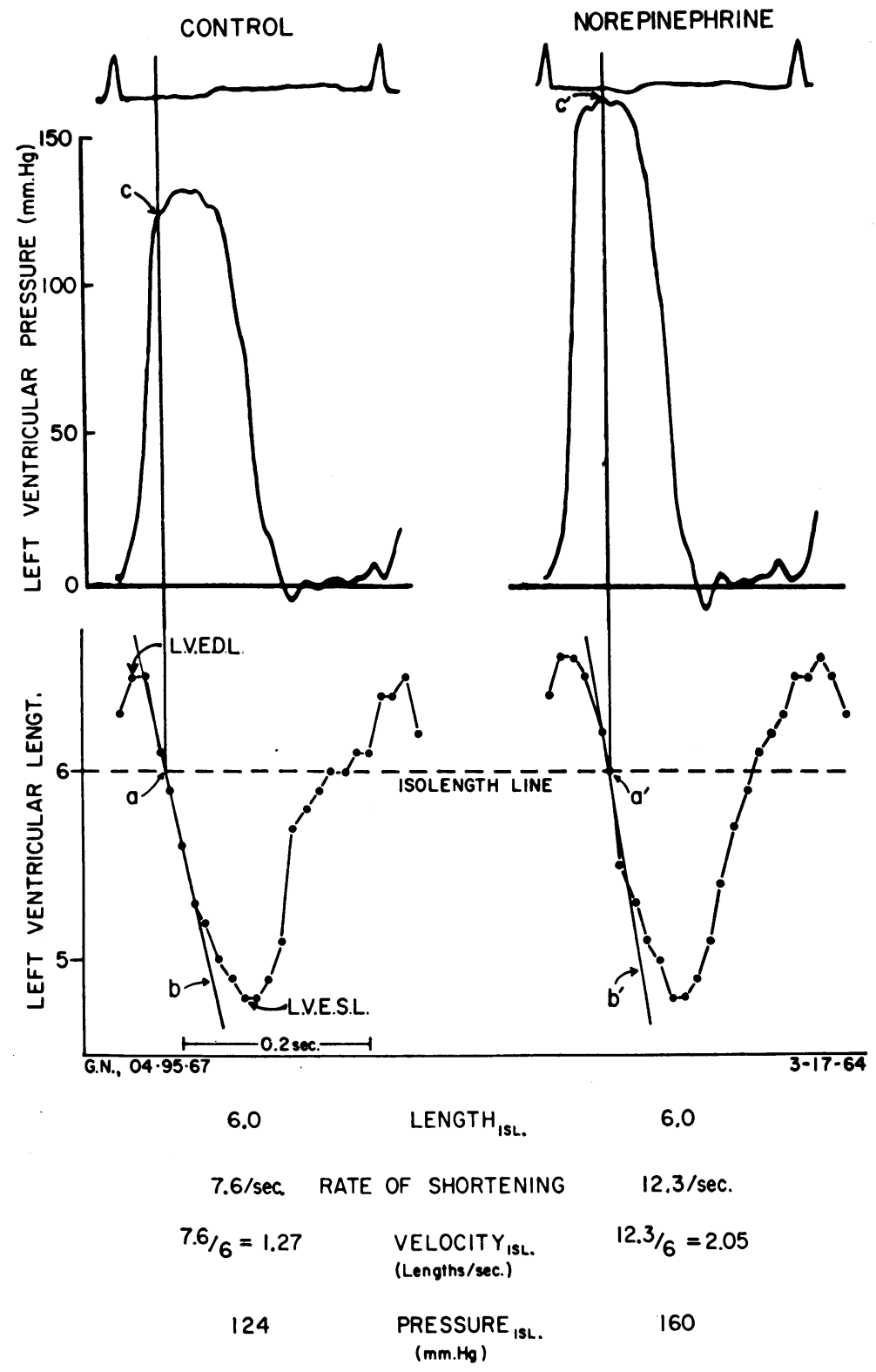

Fig. 1. Method For determining the instantaneous force-velocity RELATION ON A BEAT-to-BEAt BASIS. From above downwards are shown the electrocardiogram, the left ventricular pressure pulse, and the curve relating left ventricular dimensions, determined at $\frac{1}{30}$-second intervals, to time. L.V.E.D.L. = left ventricular end-diastolic length; L.V.E.S.L. = left ventricular end-systolic length. On the left are the data obtained during the control period and on the right the observations made during an infusion of norepinephrine. Points $a$ and $a^{\prime}$ represent the isolength points at which both instantaneous velocity of shortening and intraventricular pressure are determined. Lines $b$ and $b^{\prime}$ are the tangents to the length curves at points a and $a^{\prime}$ and represent the velocity of shortening at these points. The steeper slope of $b^{\prime}$ as compared to $b$ signifies an augmentation of velocity. Points $c$ and $c^{\prime}$ represent the temporally related points on the ventricular pressure curve. 
the tangent to the curve (Figure $1, b$ and $b^{\prime}$ ), relating ventricular length to time at this point of the curve. Although myocardial wall tension (force) could not be measured directly in these studies, it is known from LaPlace's law (12) that at any given ventricular volume, wall tension is a constant function of intraventricular pressure. Therefore, in order to utilize intraventricular pressure as a direct reflection of wall tension, the ventricular pressures that corresponded temporally to the isolength point were determined (Figure 1, points $\mathrm{c}$ and $c^{\prime}$ ).

The measurements of the distances between markers and the rate at which they aproached each other were initially expressed in centimeters, and in centimeters per second, respectively, but these measurements represent relative distances and velocities, since the absolute values are a function of the magnification that occurs when the distances between markers are photographed and projected onto a screen. However, since the degree of this magnification was constant throughout any study and analysis, it was possible in assessing velocity to eliminate the use of relative units and to express the velocity of shortening in units of "muscle lengths per second," by dividing the tangent to the shortening curve by the length between the markers at the isolength point, that is, the point at which the velocity of shortening and pressure were measured. Thus, since absolute length $=$ measured length/degree of magnification, and absolute velocity = measured velocity/degree of magnification:

measured velocity/degree of magnification measured length/degree of magnification

$$
=\frac{\text { absolute velocity }}{\text { absolute length }} \text {. }
$$

Therefore, by determining the ratio of measured velocity to measured length it was possible to express the instantaneous velocity in the commonly employed form of "muscle lengths per second" (1). In each patient, between 4 and 20 individual cycles were analyzed for each period of study.

To investigate the effects of primary increases in afterload on the force-velocity relation, methoxamine was infused intravenously into six patients in doses sufficient to raise systolic arterial pressure by an average value of 33 $\mathrm{mm} \mathrm{Hg}$. To diminish afterload, a balloon was inflated in the inferior vena cava in one patient, thereby curtailing venous return and cardiac output, as described previously
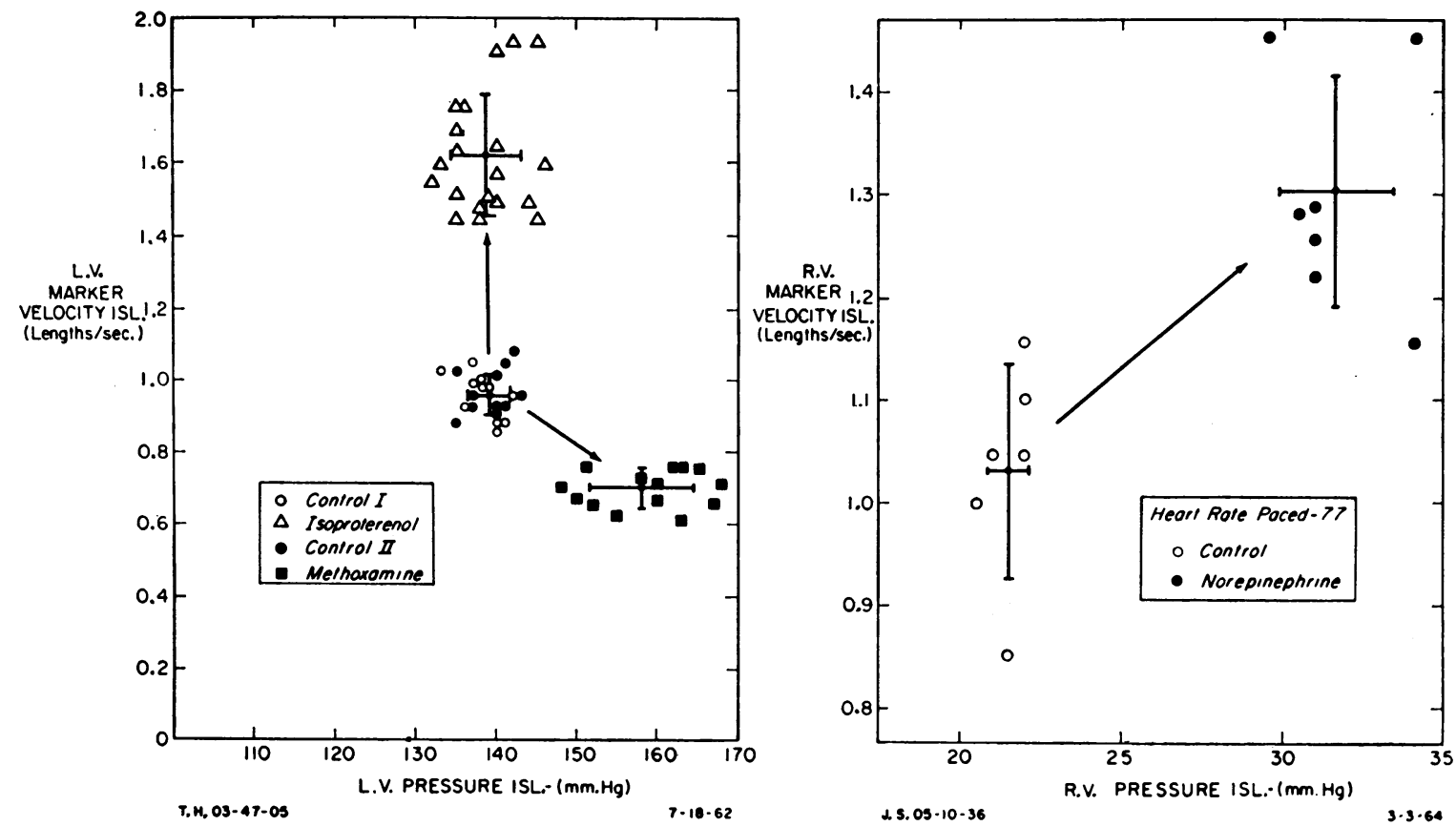

Fig. 2. LEFT PANEL: EFFeCtS OF METhOXAMine AND ISOPROTERENOL ON THE LEFT VENTRICULAR FORCE-VELOCITY RELATION. As intraventricular pressure was raised by the infusion of methoxamine, the velocity of shortening declined. However, during infusion of isoproterenol, velocity of shortening increased markedly, while intraventricular pressure did not change significantly. Velocity is $1 ~$ and pressure $_{\text {ss } 1}$ represent the velocity of shortening and the intraventricular pressure at the moment the myocardial segment length passed through the isolength point. Each symbol represents the analysis of a single cardiac cycle. In this and in subsequent figures the large crosses in each set of symbols represent the mean value and 1 SD. RIGHT PANEL: EFFEcts OF NOREPINEPHRINE INFUSION ON THE RIGHT VENTRICULAR FORCE-VELOCITY RELATION. Norepinephrine raised both pressure and velocity, i.e., the force-velocity relation was shifted upward and to the right. 


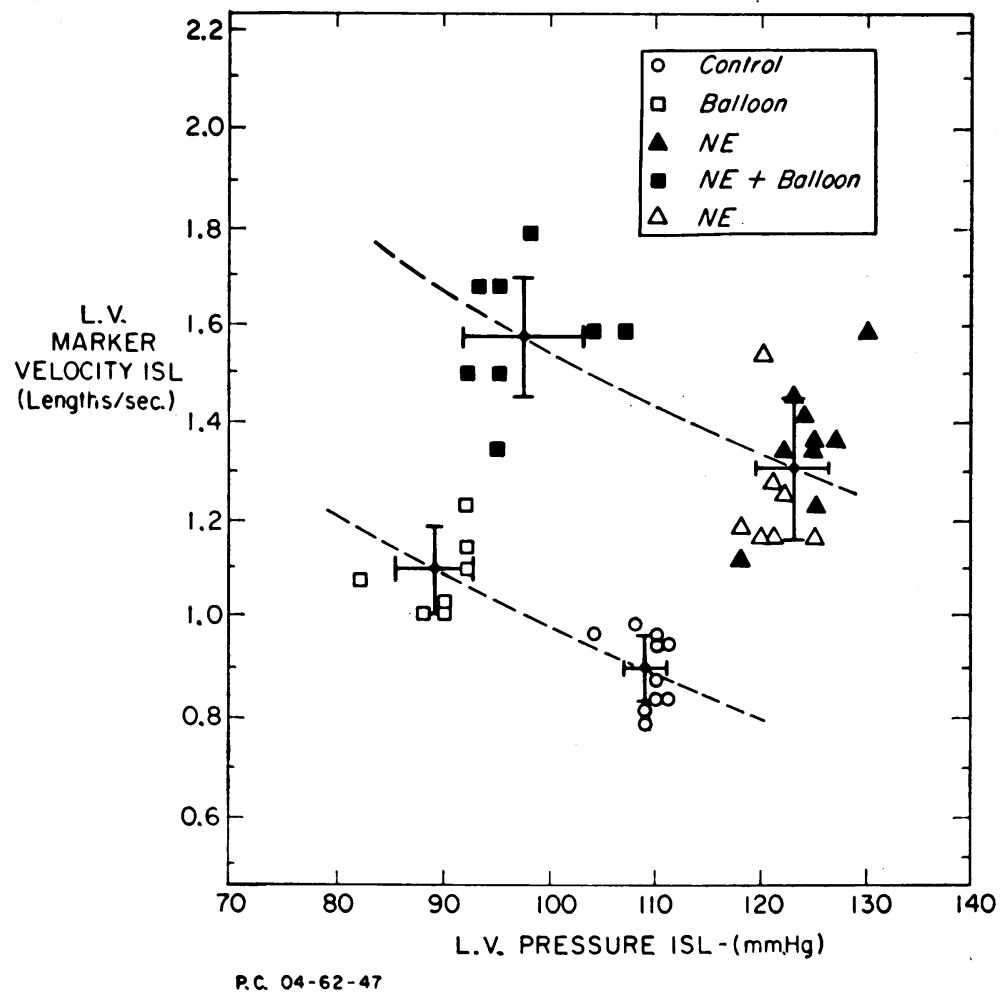

Fig. 3. COMBINED EFFECTS OF A NOREPINEPHRINE INFUSION AND DECREASING AFTERLOAD BY IMPEDING VENOUS RETURN WITH A BALLOON INFLATED IN THE INFERIOR VENA CAVA ON THE LEFT VENTRICULAR FORCE-VELOCITY RELATION. The open circles represent the data during the control period. The open squares show that when arterial pressure declined as a result of inflation of the balloon, velocity of shortening increased. The solid triangles illustrate the data obtained with the balloon deflated during norepinephrine infusion and show that the relation between force and velocity has been shifted upwards and to the right. The solid squares depict the values when the balloon has been reinflated during the course of the norepinephrine infusion and show that as afterload falls, velocity of shortening once again increases, but this time along a higher force-velocity curve. The open triangles show that when the balloon is deflated, but the norepinephrine infusion is continued, the myocardium moves back along the same force-velocity curve.

(13). A positive inotropic effect without a significant alteration of systolic arterial pressure was produced by the intravenous infusion of isoproterenol to six patients in an average dose of $2.5 \mu \mathrm{g}$ per minute. The effects of an infusion of norepinephrine in an average dose of 25 $\mu \mathrm{g}$ per minute were studied on six occasions in five patients, and in four of them the heart rate was maintained constant by means of electrical stimulation of the atrium with a bipolar electrode catheter, as described previously (14). Norepinephrine not only exerts a positive inotropic action, but also increases the afterload. To determine the effects of changes in heart rate, stepwise increases in rate were achieved in four patients by electrical stimulation of the right atrium (14). Finally, the effects on the forcevelocity relation of paired electrical stimulation, a form of sustained postextrasystolic potentiation $(15,16)$, were determined in one patient. In this technique paired electrical stimuli were delivered to the right ventricle by means of a bipolar electrode catheter inserted into the right ventricular cavity. By appropriately spacing the time interval between the paired stimuli the second impulse of each pair elicited a depolarization without a discernible second ventricular contraction.

\section{Results}

The effects of methoxamine on the force-velocity relations of the left ventricle were determined in five patients, and the results of a typical study are shown in Figure 2, left. Ventricular pressure, measured at the time of the isolength point (pres- 
TABLE II

Effects of various interventions on instantaneous myocardial force-velocity relations and on ventricular dimensions*

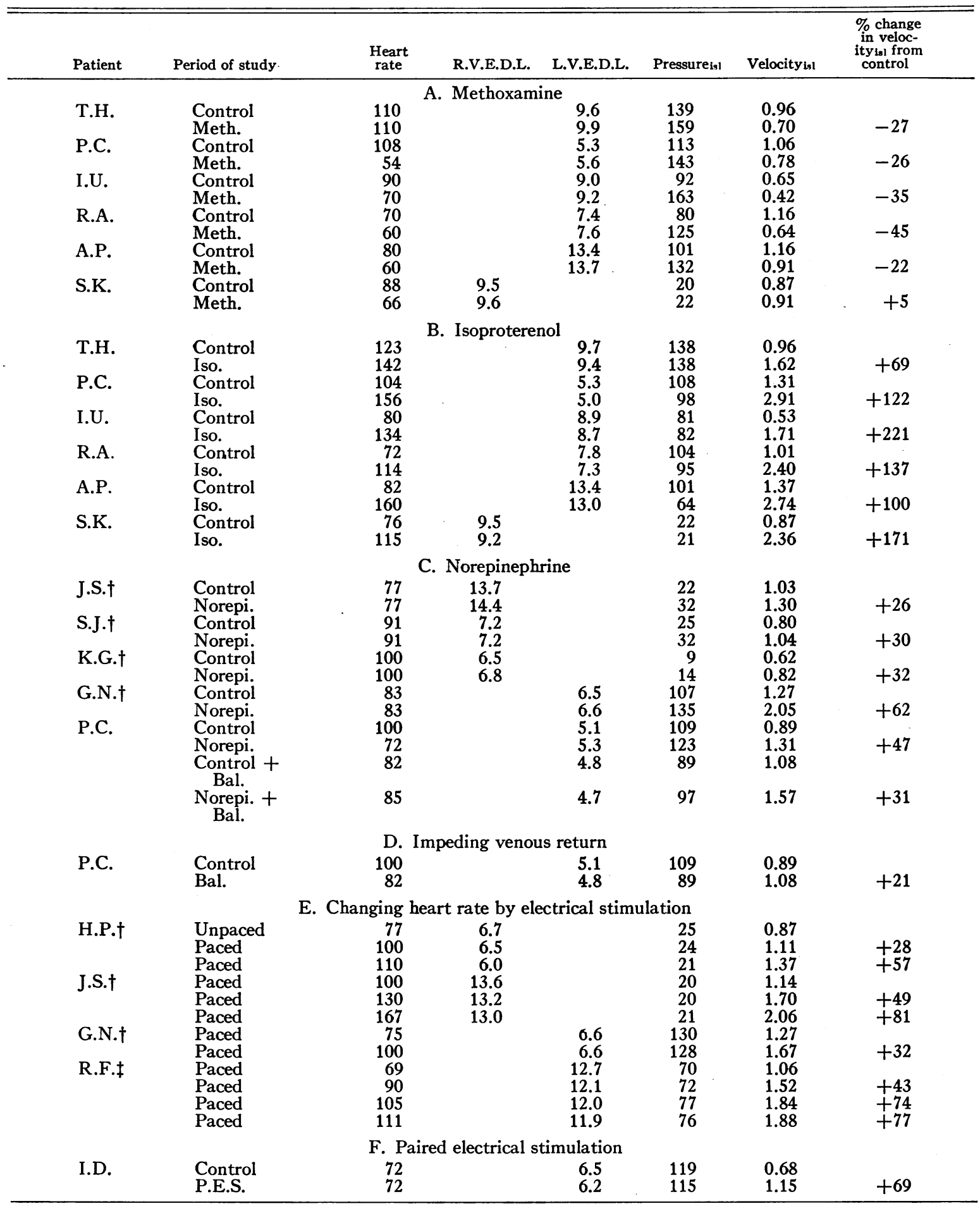

* R.V.E.D.L. = right ventricular end-diastolic length; L.V.E.D.L. = lef $t$ ventricular end-diastolic length; Pressure $=$ ventricular pressure at isolength point; Velocity ${ }_{i s l}=$ velocity of shortening at isolength point; Meth. = methoxamine infusion; Iso. = isoproterenol infusion; Norepi. = norepinephrine infusion; Bal. = balloon distended in inferior vena cava.

†.Heart paced by stimulation of right arrium by bipolar electrode catheter.

$\ddagger$ Heart paced by stimulation of left ventricle by radio-frequency pacemaker. 


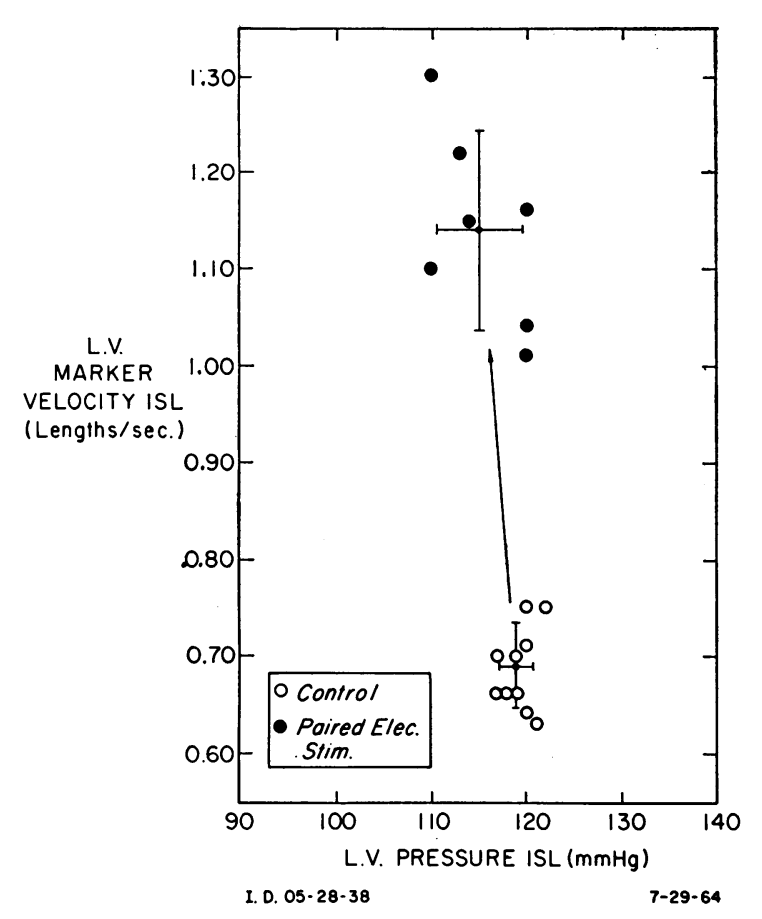

Fig. 4. EFFeCts of PAIREd Electrical stimulation ON THE LEFT VENTRICULAR FORCE-VELOCITY RELATION. Velocity of shortening increased while ventricular pressure remained essentially unchanged.

sure $\left._{\text {isl }}\right)$ rose in all five subjects, the elevation averaging $37 \mathrm{~mm} \mathrm{Hg}$ (Table II). In each of these subjects the instantaneous velocity of shortening, also measured at the isolength point (velocity $_{\text {isl }}$ ), decreased from an average of 1.00 muscle lengths per second to an average of 0.69 muscle lengths per second, representing an average decline of $31 \%$. As previously reported (17), methoxamine always increased left ventricular end-diastolic dimensions, and it decreased heart rate in four of the five patients. The effects of methoxamine on the force-velocity relations of the right ventricle were studied in one patient (S.K.) ; no significant alteration in the systolic pressure or velocity of shortening occurred in her.

Inflating a balloon in the inferior vena cava resulted in a decline in left ventricular pressure isl $_{\text {in }}$ from 109 to $89 \mathrm{~mm} \mathrm{Hg}$ and a reciprocal increase in velocity $\mathrm{isl}_{\mathrm{il}}$ of $21 \%$ (Table II, P.C.) (Figure 3 ).

The effects of isoproterenol on the force-velocity relation of the left ventricle were determined in five patients and on the right ventricle in one patient. Ventricular pressure isl $_{\text {exhibited little }}$ change in five patients and declined significantly in one (A.P.). Velocity ${ }_{\text {isl }}$, however, increased in every patient, from an average value of 1.01 to 2.29 muscle lengths per second, representing an average rise of $137 \%$ (Figure 2, left). These alterations were associated with an increase in heart rate and a decline in ventricular end-diastolic dimensions (17).

Paired electrical stimulation, resulting in a ventricular contraction rate which was identical to that observed during sinus rhythm, exerted an effect similar to that of isoproterenol, in that velocity $_{\text {isl }}$ increased markedly, ventricular enddiastolic length decreased, and ventricular pressure $_{\text {isl }}$ remained constant (Figure 4).

The effects of norepinephrine were determined in six studies on five patients; three studies were performed on each ventricle. The results were directionally similar in all six studies, whether

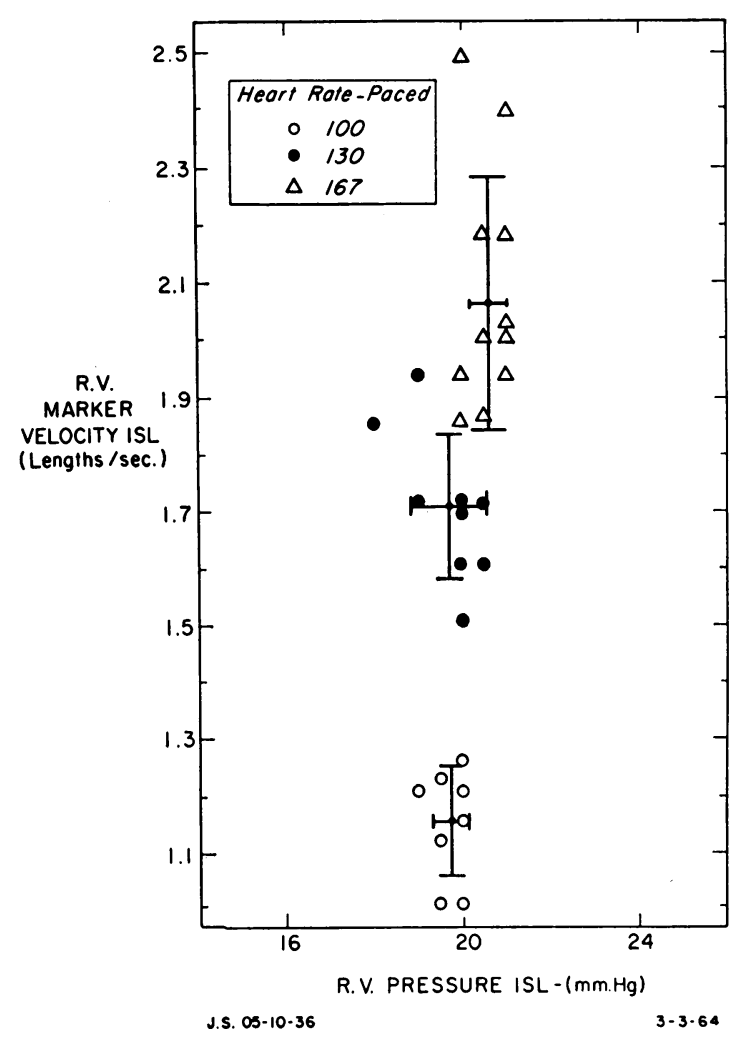

Fig. 5. EFFECTS OF INCREASING heart RATE by STIMULATION OF THE RIGHT ATRIUM WITH A BIPOLAR ELECTRODE CATHETER ON THE RIGHT VENTRICULAR FORCE-VELOCITY RELATION. As heart rate was elevated in stepwise fashion, the velocity of shortening increased progressively, while pressure was unaltered, indicating augmentation of the contractile state of the myocardium. 
heart rate was controlled or not. As observed with methoxamine, but not as noted with isoproterenol, an increase in pressure ${ }_{\text {isl }}$ occurred (Figure 2, right). On the other hand, the effects of norepinephrine on the velocity ${ }_{\text {isl }}$ were directionally similar to those resulting from isoproterenol, velocity $_{\text {isl }}$ rising from an average of 0.95 to 1.35 muscle lengths per second, representing an average increase of $38 \%$.

The effects of raising heart rate by electrical stimulation resulted in only slight changes in ventricular pressure in all four patients. In each instance velocity isl $_{\text {increased, from an average of }}$ 1.08 muscle lengths per second at an average heart raté of 80 to an average of 1.75 muscle lengths per second at an average heart rate of 122 , equivalent to an average rise of $61 \%$ (Figure 5). These changes were accompanied by a decline of ventricular end-diastolic length in three studies and no change in the fourth (18).

The combined effects of impeding venous return and norepinephrine infusion in patient P.C. are illustrated in Figure 3 . Although inflating the balloon lowered left ventricular pressure isl and raised velocity ${ }_{i s l}$, both before and during norepinephrine infusion, the relationship between the two variables was displaced upwards and to the right by the norepinephrine infusion.

\section{Discussion}

Since the cineradiographic technique employed in this investigation allows measurement of the relative distances between two markers on the external ventricular surface at intervals of $\frac{1}{30}$ second, it may also be used to determine the rate of shortening of the segment of ventricular wall between these markers. Although the calculations utilized permitted evaluation of the force-velocity relation of only a segment of myocardium, the available evidence suggests that the contractile state of a segment of a ventricle is representative of the entire ventricle (19). Furthermore, in other studies, it has been shown that during ventricular ejection the shear stresses through the myocardial wall are small, that is, little sliding of various muscle layers over one another occurs, and therefore data obtained from markers attached to the surface of the ventricle would appear to be valid representations of relative changes in muscle length throughout the myocardial wall (20). Since it is possible to apply the roentgenopaque markers to the human heart only when it is already exposed in the course of a corrective operation, none of the subjects in whom this study was carried out had entirely normal hearts. However, the surgical procedure had, in each instance, corrected the mechanical defect, and it is likely that the responses of truly normal hearts are similar to those that were noted in this investigation.

Most of the interventions employed resulted in changes in ventricular end-diastolic dimensions, and it is now well known that the myocardial force-velocity relation is changed profoundly by alterations in the initial length of the muscle $(3,4)$. However, it has recently been shown in the isolated cat papillary muscle that, for any given contractile state of the myocardium, the force-velocity relation is a function of the instantaneous length at which the relationship is determined and is essentially independent of the diastolic length from which the contraction is initiated (11). The fundamental importance of using instantaneous length in the characterization of ventricular force-velocity relations has also been recognized by Fry, Griggs, and Greenfield (7). Since myocardial force is a function of intraventricular pressure and size, by always determining pressure at the same ventricular dimensions in a given study, changes in pressure could be used to indicate changes in force. Finally, the measurement of both velocity of shortening and intraventricular pressure as the myocardial segment length passed through the isolength point made it possible to determine the effects of a number of different interventions on the force-velocity relation. It is also recognized that the velocity of myocardial fiber shortening is not equal to the velocity of shortening of the contractile elements except when the series elastic component is not changing in length (8). However, the extension of the series elastic takes place almost entirely during the isovolumetric phase of contraction and is virtually complete at the point during the ejection phase at which the measurements were made. Thus, it is unlikely that alterations of the rate of the elongation of the series elastic could have altered the relationship between the velocity of 
shortening of the segment of myocardium and of the contractile elements.

It was observed that when ventricular afterload was augmented with methoxamine or was decreased by inflating a balloon in the inferior vena cava, the resultant changes in pressure and velocity varied reciprocally (Figure $6, \mathrm{~A}-\mathrm{B}$ and $\mathrm{A}-\mathrm{B}^{\prime}$ ). Thus, when afterload is altered, the myocardium is moved along a force-velocity curve that characterizes the contractile state of the myocardium under the specific conditions existing at the time of study. Methoxamine was the drug selected to augment ventricular afterload since this pressor amine has been shown to have no direct action on the myocardium $(21,22)$. Indeed, methoxamine was found to have no effect on the relation between pressure and velocity of the right ventricle of patient S.K., further substantiating the notion that the changes in the pressure-velocity relation observed in the left ventricle resulted primarily from a change in afterload. These find-

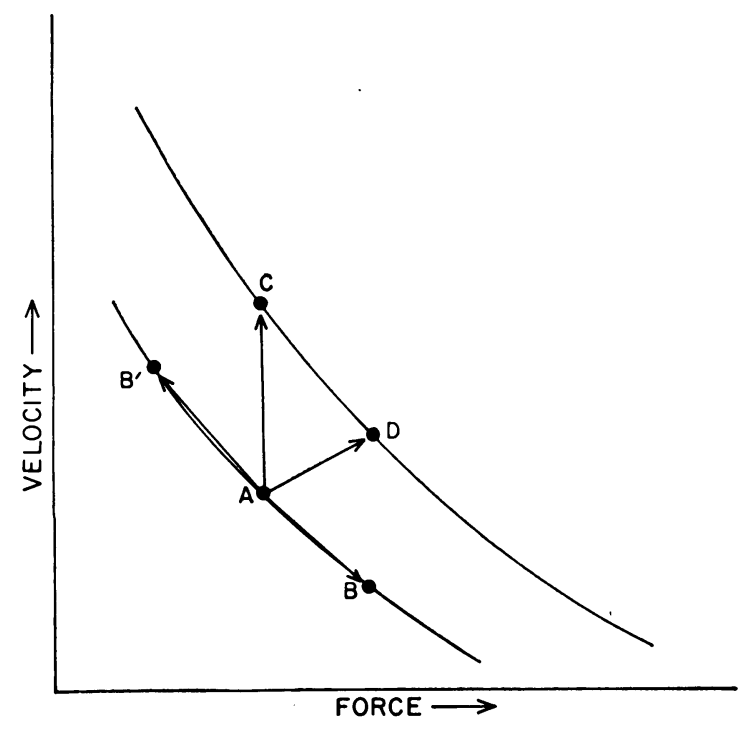

Fig. 6. Diagrammatic Representation of the EFFECTS OF VARIOUS INTERVENTIONS ON THE MYOCARDIAL FORCE-VElocity Relation. Point A represents the control values for force and velocity. An increase or a decrease in afterload results in a reciprocal change in velocity, with movement along the lower curve to points B and $\mathrm{B}^{\prime}$, respectively, indicating no change in the contractile state of the myocardium. A pure inotropic intervention shifts the force-velocity relation to point $C$, while an intervention that produces both an increase in afterload and a positive inotropic effect shifts the relation to point $D$. ings in the intact heart are entirely analogous to the inverse relationship between force and velocity observed in isolated skeletal and in heart muscle $(1-8,11)$. Since heart rate usually diminished somewhat while arterial pressure rose, it is possible that some of the decrease in velocity of shortening that was observed with this drug resulted from these changes in heart rate. It is unlikely that the slowing played an important role, since, in all but one patient (P.C.), the degree of slowing produced by methoxamine was relatively small, and in patient T.H., in whom no changes in rate occurred, the aforementioned reciprocal relation between pressure and velocity of shortening was observed.

In contrast to the results obtained when afterload was altered while the contractile state of the myocardium remained constant, the administration of isoproterenol increased the velocity of shortening without usually altering ventricular pressure significantly. From studies on isolated muscle, it is known that this catecholamine augments the contractile state of the myocardium (23). The increase in velocity of shortening at a constant ventricular pressure (Figure $6, \mathrm{~A}$ to C) is considered to represent a shift of the forcevelocity curve and indicates that the myocardial contractile state was improved by the drug in these patients.

It has been shown in the isolated cat papillary muscle that an increase in the frequency of contraction produces an increase in the velocity of shortening at any given load $(2,3)$. Similarly, in intact conscious patients, augmenting heart rate had the same effect (Figure 5), which, although of smaller magnitude than that observed with the administration of isoproterenol, also represents a positive inotropic intervention. These considerations make it clear that part of the increase in the velocity of shortening noted during isoproterenol administration resulted from the concomitant elevation of heart rate.

The effects of paired electrical stimulation, a form of sustained extrasystolic potentiation, were studied in one patient. This intervention, which has previously been shown to augment the contractile state of the myocardium, both in isolated cardiac tissue (16) and in the intact canine heart (24), was shown in this investigation to result in a shift of the force-velocity relation in a man- 
ner analogous to that observed when isoproterenol was given or when heart rate was increased.

The effects of norepinephrine on the isolated papillary muscle have been studied extensively, and this agent has been shown to increase both the maximal force and velocity (3). However, in the intact organism this drug also increases the afterload imposed on the ventricle, primarily by increasing systemic vascular resistance. Thus, as a consequence of its stimulating effect on both the alpha and beta adrenergic receptors, norepinephrine simultaneously increased pressure and augmented the velocity of shortening (Figure 6, A to D). Furthermore, to prevent reflex changes in heart rate that might, by themselves, alter the force-velocity relation, heart rate was maintained constant in four of the six studies. The effect of norepinephrine on the force-velocity curve is exemplified by the study in patient P.C. in whom the effect of changes in afterload was studied both before and during the infusion of this agent. Diminishing afterload increased velocity of shortening, regardless of the catecholamine background to which the heart was exposed, but it is apparent that during the norepinephrine infusion the heart operates on a higher forcevelocity curve (Figure 3 ).

In conclusion, the present investigation demonstrates that the force-velocity relationship, which characterizes the active contractile elements of muscle (1), has a valid counterpart not only in isolated human heart muscle (6), but in the functioning ventricle of intact, human subjects as well. In skeletal muscle, the rate with which energy leaves the active muscle is governed by this basic relation (1) attesting to the close link between the mechanics of contraction and the chemical reactions which generate force (25). Whereas in skeletal muscle the force-velocity relation is unique, save for changes in temperature (1), the force-velocity relation may be shifted in heart muscle by inotropic interventions. This shift in the force-velocity curve is always characterized by an increase in the maximal velocity of shortening whether or not a change in maximal force occurs and serves to define the contractile state of the muscle. The present study demonstrates that the effects of a variety of common physiologic and pharmacologic interventions on the function of the heart in intact man can be expressed in terms of the manner in which these interventions affect the underlying muscle mechanics. Furthermore, it now also appears feasible to compare the contractile state of the myocardium in the same patients at various stages of their disease and to compare the force-velocity relation among different patients.

\section{Summary}

With a cineradiographic technique, myocardial force-velocity relations were investigated in 23 studies on 13 patients at the time of postoperative cardiac catheterization. The technique consisted of exposing cineradiograms at 30 frames per second and measuring the velocity of movement of roentgenopaque markers that had been sutured to the external surface of the ventricles, while simultaneously recording intraventricular pressure. A beat-to-beat analysis of the force-velocity relationship was then accomplished by measuring the velocity and the pressure at a constant length point in each contraction. Thereby, a constant relation was established between intraventricular pressure and myocardial wall tension. When afterload was augmented with methoxamine or was decreased by impeding venous return with a balloon distended in the inferior vena cava, force and velocity varied inversely. In contrast, norepinephrine, isoproterenol, increasing heart rate by electrical pacing, and paired electrical stimulation all augmented velocity at any given pressure. Thus, in intact, conscious man, the heart displays the same reciprocal relation between velocity of shortening and generation of force observed in isolated papillary muscle, and a change in the contractile state of the human heart is manifested by a shift in the force-velocity relation.

\section{References}

1. Hill, A. V. The heat of shortening and the dynamic constants of muscle. Proc. roy. Soc. B 1938, 126, 136.

2. Abbott, B. C., and W. F. H. M. Mommaerts. A study of inotropic mechanisms in the papillary preparation. J. gen. Physiol. 1959, 42, 533.

3. Sonnenblick, E. H. Force-velocity relations in mammalian heart muscle. Amer. J. Physiol. 1962, 202, 931.

4. Sonnenblick, E. H. Implications of muscle mechanics in the heart. Fed. Proc. 1962, 21, 975. 
5. Sonnenblick, E. H. Series elastic and contractile elements in heart muscle: changes in muscle length. Amer. J. Physiol. 1964, 207, 1330.

6. Sonnenblick, E. H., E. Braunwald, and A. G. Morrow. The contractile properties of human heart muscle: studies on myocardial mechanics of surgically excised papillary muscles. J. clin. Invest. 1965, 44, 966.

7. Fry, D. L., D. M. Griggs, Jr., and J. G. Greenfield, Jr. Myocardial mechanics: tension-velocity-length relationships of heart muscle. Circulat. Res. 1964, 14, 73.

8. Levine, H. J., and N. A. Britman. Force-velocity relations in the intact dog heart. J. clin. Invest. 1964, 43, 1383.

9. Harrison, D. C., A. Goldblatt, and E. Braunwald. Studies on cardiac dimensions in intact, unanesthetized man. I. Description of techniques and their validation. Circulat. Res. 1963, 13, 448.

10. Goldblatt, A., D. C. Harrison, G. Glick, and E. Braunwald. Studies on cardiac dimensions in intact, unanesthetized man. II. Effects of respiration. Circulat. Res. 1963, 13, 455.

11. Sonnenblick, E. H. Instantaneous force-velocitylength determinants in the contraction of heart muscle. Circulat. Res. 1965, 16, 441.

12. Burton, A. C. The importance of the shape and size of the heart. Amer. Heart J. 1957, 54, 801.

13. Ross, J., Jr., and E. Braunwald. Studies on Starling's law of the heart IX: The effects of impeding venous return on performance of the normal and failing human left ventricle. Circulation 1964, 30, 719.

14. Ross, J., Jr., J. W. Linhart, and E. Braunwald. Effects of altering heart rate by electrical stimulation of the right atrium in man: studies at rest, during muscular exercise and isoproterenol infusion. Circulation, in press.

15. Braunwald, E., J. Ross, Jr., P. L. Frommer, J. F. Williams, Jr., E. H. Sonnenblick, and J. H. Gault. Clinical observations on paired electrical stimulation of the heart. Effects on ventricular perform- ance and heart rate. Amer. J. Med. 1964, 37, 700.

16. Kruta, V., and P. Braveny. Restitution de la contractilité du myocarde entre les contractions et les phénomènes de potentiation. Arch. intern. Physiol. 1961, 69, 645.

17. Harrison, D. C., G. Glick, A. Goldblatt, and E. Braunwald. Studies on cardiac dimensions in intact, unanesthetized man. IV. Effects of isoproterenol and methoxamine. Circulation 1964, 29, 186.

18. Glick, G., J. F. Williams, Jr., D. C. Harrison, A. G. Morrow, and E. Braunwald. Studies on cardiac dimensions in intact, unanesthetized man. V. Effects of changes in heart rate produced by electrical stimulation. Submitted for publication.

19. Cotten, M. deV. Circulatory changes affecting measurement of heart force in situ with strain gauge arches. Amer. J. Physiol. 1953, 174, 365.

20. Feigl, E. O., and D. L. Fry. Intramural myocardial shear during the cardiac cycle. Circulat. Res. 1964, 14, 536.

21. Goldberg, L. I., M. deV Cotten, T. D. Darby, and E. V. Howell. Comparative heart contractile force effects of equipressor doses of several sympathomimetic amines. J. Pharmacol. exp. Ther. 1953, 108, 177.

22. Goldberg, L. I., R. D. Bloodwell, E. Braunwald, and A. G. Morrow. The direct effect of norepinephrine epinephrine and methoxamine on myocardial contractile force in man. Circulation 1960, 22, 927.

23. Garb, S. Inotropic action of epinephrine, norepinephrine and $\mathrm{N}$-isopropyl norepinephrine on heart muscle. Proc. Soc. exp. Biol. (N. Y.) 1950, 73, 1950.

24. Ross, J., Jr., E. H. Sonnenblick, G. A. Kaiser, P. L. Frommer, and E. Braunwald. Electroaugmentation of ventricular performance and oxygen consumption by repetitive application of paired electrical stimuli. Circulat. Res. 1965, 16, 332.

25. Podolsky, R. J. Mechanochemical basis of muscular contraction. Fed. Proc. 1962, 21, 964. 